\title{
Continuous Intravenous Infusion
}

National Cancer Institute

\section{Source}

National Cancer Institute. Continuous Intravenous Infusion. NCI Thesaurus. Code C38196.

A route of administration of a fluid drug form into a vein or veins, over a time duration of equal to or more than 24 hours. Method allows to maintain steady state plasma/serum medication levels, a smaller total daily dose of drug to achieve the same pharmacodynamic endpoint in comparison with intermittent or bolus infusion, and may result in lower toxicity. The method has a positive impact on health-care costs. 